\title{
The Case for Researching Biomechanics and Human Movement Using a Stochastic Model in a Peer Reviewed Journal to Generate Qualitative Evidence
}

\section{ISSN: 2637-8078}

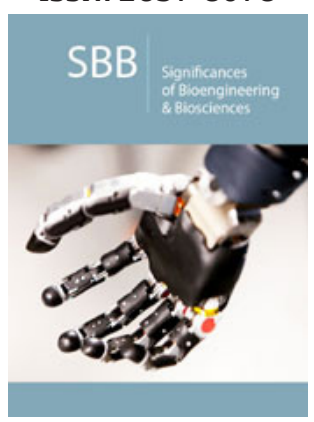

*Corresponding author: Shavelson D, Department of surgery, USA

Submission: 㥁 March 18, 2019

Published: 傋April 22, 2019

Volume 3 - Issue 1

How to cite this article: Shavelson D, The Case for Researching Biomechanics and Human Movement Using a Stochastic Model in a Peer Reviewed Journal to Generate Qualitative Evidence.Significances Bioeng Biosci.3(1). SBB.000553.2019.

DOI: 10.31031/SBB.2019.03.000553.

Copyright@ Shavelson D, This article is distributed under the terms of the Creative Commons Attribution 4.0 International License, which permits unrestricted use and redistribution provided that the original author and source are credited.

\section{Shavelson D*}

Department of surgery, USA

\section{The Problem}

The Evidence Based Practice (EBP) of biomechanics and human movement (hereafter BHM) has been handcuffed by a lack of peer reviewed, high level, valid and clinically applicable evidence that has dampened the ability to use Sackett's model of Evidence Based Medicine (EBM) clinically [1-3]. Current journal research protocols for EBM follow a deterministic model. In this model there are one or two dependent variables to be investigated and simultaneously all independent variables that could dampen the results are eliminated or controlled with an algorithm or statistical formulae. The deterministic model has both a high intratester (the same tester repeatedly gets the same results) and intertester (different testers repeatedly get the same results) efficiency a for it to be deemed high level. Because of the vast numbers of independent variables that exist when researching BHM [4], high level research has been forced to be complaint oriented (limited to signs or symptoms such as pain, an injury or a deformity [5]reducing its ability to have clinical applicability on a case to case basis.

Valid longitudinal studies in biomechanics have been attempted but few make Sackett's high levels of evidence criteria due to changes in independent variables over time that cannot be controlled or eliminated. One of these few, The Framingham Study [6] is so old and outdated that its statistics are being called obsolete. Current EBM models cannot and have not investigated prevention, performance enhancement and quality of life upgrades (Salutogenetic dependent variables) leaving clinicians waiting for a clinical complaint to justify seeing patients instead of diagnosing and treating underpinning precursor probabilities before, during or after a complaint in an integrative, holistic sense. Summarily, a failure to isolate the controlled variables or to conduct any study long term, in any experimental design seriously compromises the internal validity of any deterministic project ruining the experiment, wasting time and resources, and damaging the researcher's reputation [7]. Stochastic models, on the other hand possess some inherent randomness and accepted independent variables as exist in nature and life. The same set of parameter values and initial conditions will lead to an ensemble of different outputs. The natural world is buffeted by stochasticity. Stochastic models are considerably more qualitative than quantitative. Stochastic biomechanical modeling is an effective and efficient research method for investigating the random outcomes of human movement. This method allows investigators to determine the risk for an injury without following subjects to obtain actual injury cases.

This enables the execution of the study to be less complicated, less labor intensive, less time consuming, and less expensive in comparison to traditional methods. Also, stochastic biomechanical modeling methods allow investigators to identify risk factors with causeand-effect relationships to the injury in the absence of any observed injuries. Stochastic 
biomechanical modeling method has been successfully applied to studies on the variation of human movements and prevention of a variety of musculoskeletal system injuries [8]. Admittedly, stochastic models have a lower level of dependability and predictability than their deterministic cousins, but they have clinical value when measurable, reproducible and clinically applicable peer reviewed evidence simply does not exist. The probability distributions of qualitative models offer starting platforms to diagnosis and treatment BHM on a case to case basis that depending on the education, skill level and expertise of the treating medical professional or therapist leads to better diagnosis and better treatment planning and implementation clinically then an absence of evidence [8].

\section{The Solution}

As I practice and research lower extremity biomechanics, architecture and body kinetics and kinematics in stance and moving when weighted, the only method of research that can produce longitudinal, viable and applicable clinical roadmaps for care is often Stochastic. I submit that cohorts can be developed with inherited, anatomical and systematic traits such as foot type, limb length inequality or excessive and limited joint ranges of motion can, in the face of independent variables on a case to case basis, can provide a body of stochastic evidence that can fill the void that exists in the peer reviewed literature when dealing with complex biomechanical and human movement issues like the foot and posture in closed chain with valid and applicable tools. My take is that we need a new journal that peer reviews stochastic, well planned and executed qualitative study of subjects like BHM as scientifically and evidence based as possible. I suggest The Journal of Closed Chain Structure and Function of the Foot \& Posture.

\section{The Foot Centering Typing System}

As an example, and with bias as I am its inventor, the foot centering foot typing system [9] classifies all feet into five common foot types each with its own characteristic weaknesses and strengths [10]. Supinatory and pronatory positions of the end ranges of motion of the critical closed chain joints of the rearfoot and forefoot are measured qualitatively on their dominant anatomical planes (the frontal plane for the rearfoot and the sagittal plane of the forefoot) and the total range of motion between the supinatory end range of motion (SERM) and the pronatory end range of motion are qualitatively measured as low, moderate or high. Treatment is designed to reduce unwanted positioning in closed chain in the rearfoot and forefoot and to reduce biomechanically pernicious ranges of high and low motion in the rearfoot and forefoot, foot type specific, regardless of independent variables. The foot centering foot typing system has high intratester and high intertester testing results. Foot typing allows for the development of risk factors as well as preventive, performance enhancing and quality of life upgrading when researching biomechanics and human movement because maintaining more optimal function position and ranges of rearfoot and forefoot end ranges of motion drives stability, support, strength, symmetry and balance to the lower extremity and the posture in closed chain.

\section{References}

1. Shavelson D (2010) Biomechanics EBM part I: Where does biomechanics EBM stand. The Foot in Closed Chain, Present Podiatry E-zine.

2. Shavelson D (2010) Biomechanics EBM part II: Biomechanics evidence-based practice (EBP). The Foot in Closed Chain, Present Podiatry E-zine.

3. Shavelson D (2010) Biomechanics EBM part III: The levels of evidence in EBM/EBP. The Foot in Closed Chain, Present Podiatry E-zine.

4. Shavelson D (2018) Resistance training (RT): A meta-analysis of the existing EBM. EC Orthopedics 9(7): 434-457.

5. De Schepper J, Van Alsenoy K, Roosen P, Rijckaert J, De Mits S, et al. (2012) Intratest reliability in determining the subtalar joint axis using the palpation technique described by K. Kirby. J Am Podiatr Med Assoc 102(2): 122-129.

6. Galica AM, Hagedorn TJ, Hillstrom HJ, Dufour AB, Riskowski JL, et al. (2013) Hallux valgus and plantar pressure loading: The framingham foot study. J foot Ankle Res 6(1): 42.

7. Henrickson D (2010) Research methods: Planning variables, British columbia library open ended texts.

8. Lin CF, Gross M, Ji C, Padua D, Weinhold P, et al. (2009) A stochastic biomechanical model for risk and risk factors of non-contact anterior cruciate ligament injuries. J Biomech 12:1-6.

9. Shmulevich I, Aitchison JD (2009) Deterministic and stochastic models of genetic regulatory networks. Methods Enzymol 467: 335-356.

10. Shavelson D (2007) A Closer look at neoteric biomechanics. Podiatry Today 20(9): 60-66. 